\title{
Lane-Change Fuzzy Control in Autonomous Vehicles for the Overtaking Maneuver
}

\author{
José E. Naranjo, Carlos González, Member, IEEE, Ricardo García, and Teresa de Pedro
}

\begin{abstract}
The automation of the overtaking maneuver is considered to be one of the toughest challenges in the development of autonomous vehicles. This operation involves two vehicles (the overtaking and the overtaken) cooperatively driving, as well as the surveillance of any other vehicles that are involved in the maneuver. This operation consists of two lane changes-one from the right to the left lane of the road, and the other is to return to the right lane after passing. Lane-change maneuvers have been used to move into or out of a circulation lane or platoon; however, overtaking operations have not received much coverage in the literature. In this paper, we present an overtaking system for autonomous vehicles equipped with path-tracking and lane-change capabilities. The system uses fuzzy controllers that mimic human behavior and reactions during overtaking maneuvers. The system is based on the information that is supplied by a high-precision Global Positioning System and a wireless network environment. It is able to drive an automated vehicle and overtake a second vehicle that is driving in the same lane of the road.
\end{abstract}

Index Terms-Fuzzy control, hybrid control, intelligent control, proportional-integral differential (PID) control, road vehicle control.

\section{INTRODUCTION}

$\mathbf{T}$ HE LANE-CHANGE maneuver is one of the most thoroughly investigated automatic driving operations for autonomous vehicles after trajectory tracking. This maneuver is used as a primitive for performing more complex operations like changing lanes on a highway, leaving the road, or overtaking another vehicle on a two-way road. In this paper, we will focus on this third maneuver because it is one of the less wellresearched issues in the autonomous vehicle field.

Manuscript received November 6, 2006; revised June 5, 2007, December 17, 2007, and December 24, 2007. This work was supported in part by the Spanish Ministry of Education under Grant Centro de Investigación Científica y Tecnológica DPI2005-07980-C03-01, in part by the Spanish Ministry of Public Works, and in part by the Citroën España S.A. under Contract "Adquirir nuevos conocimientos sobre la introducción de las tecnologías de la información en el mundo del automóvil y para difundirlos en los ámbitos científicos, empresariales y comerciales (AUTOPIA)," European Union-Specific Targeted Research Project CyberCars2. The Associate Editor for this paper was D. Zheng.

J. E. Naranjo was with the Instituto de Automática Industrial (CSIC), 28500 Madrid, Spain. He is now with the Department of Intelligent Systems, Escuela Universitaria de Informática, Universidad Politécnica de Madrid, 28031 Madrid, Spain (e-mail: joseeugenio.naranjo@upm.es; jnaranjo@eui.upm.es).

C. González, R. García, and T. de Pedro are with the Industrial Computer Science Department, Instituto de Automa'tica Industrial (CSIC), 28500 Madrid, Spain (e-mail: gonzalez@iai.csic.es; ricardo@iai.csic.es; tere@iai.csic.es).

Color versions of one or more of the figures in this paper are available online at http://ieeexplore.ieee.org.

Digital Object Identifier 10.1109/TITS.2008.922880
A total of 13939 fatal crashes occurred during overtaking maneuvers in the United States from 1994 to 2005. As a direct consequence of these accidents, 24565 people died (Fatality Analysis Reporting System, National Center for Statistics and Analysis, U.S. Department of Transportation). Most of these accidents were caused by failing to leave enough distance, overtaking when there was poor visibility, or by not giving way to an overtaking vehicle.

Consequently, First World governments are under pressure to take action to reduce such a high number of fatalities. Perhaps one of the most drastic actions is the measure taken by the government of The Netherlands. It has banned overtaking on two-way roads, which is an inconvenience for drivers [1]. The objective of driving assistance and autonomous driving system research is to avoid this kind of Draconian measure, as well as to improve the safety and comfort of potentially dangerous maneuvers like overtaking.

One basic element in autonomous vehicle development is lateral or automatic steering management. According to the literature, the first automated steering wheel was built in 1977 in Japan as part of the Comprehensive Automobile Traffic Control System project under the direction of Prof. Tsugawa [2]. As part of the ARGO project [3], which was developed at Parma University by Prof. Broggi's team, a vehicle was instrumented with artificial vision cameras and a PC-based computer to automatically manage the steering wheel on routes along public highways. The guidance system is based on a classical P controller, whose input signals are directly supplied by the lane recognition vision system [4]. Carnegie Mellon University's NavLab laboratory has gained a lot of experience in developing steering controllers for the NavLab vehicle series. These vehicles are equipped with artificial vision systems, and the steering of the early versions was controlled by the neural-network-based Rapidly Adapting Lateral Position Handler (RALPH) [5]. Several lateral controllers have also been developed at the Partners for Advanced Transit and Highways (PATH) Program [6]-[9]. One such controller is described in [10], where Hessburg and Tomizuka presented a fuzzy-controlbased lateral vehicle guidance system that has been installed in a Toyota Celica experimental test vehicle. There are other techniques for controlling steering, such as $\mathrm{H} \infty$, Adaptive, and PID, as described by Chaib et al. [11]. Other real vehicle applications have been developed that are capable of autonomous steering management and performing human-like tracking [12]-[14].

After the steering wheel of a vehicle has been automated, it can do more complex maneuvers that may require high-level 
planning and information-sharing cooperation among vehicles that are circulating along the same stretch of the road. Automatic parking [15], obstacle avoidance, intersection automatic management, and overtaking, which is the maneuver studied in this paper, are some examples of this kind of cooperative autonomous driving.

There is a lot of literature, as well as research projects, related to the lane-change issue, which has mainly been generated by the California PATH Program. In [6], a lane change is used to get an autonomous vehicle to automatically leave or join a platoon of unmanned vehicles circulating in a different lane. This paper describes a control system for lane keeping and lane change. Lane change is carried out by defining the estimated lane-change trajectory and then using onboard sensors to track the path. It is a three-phase maneuver-lane exit, new lane entry, and lane tracking. A classic analytical control system is proposed. Its input variables are lateral and angular errors from the magnetic marker sequence that is placed in the center of the lane. Other PATH groups are working on lane-change systems using diverse techniques. Hessburg and Tomizuka [16] from the Department of Mechanical Engineering, University of California, Berkeley, presented a fuzzy controller to manage the steering wheel of an autonomous vehicle in lane-change maneuvers. The rule base of this system consists of 24 linguistic rules, three control inputs (the lateral lane displacement, the lateral acceleration, and the lateral acceleration error), and one output (the steering target angle).

Another PATH application using the automatic lane-change maneuver is as a response to an emergency situation like obstacle avoidance. Swaroop and Yoon [17] from Texas A\&M University, College Station, presented the design of a controller, control law, and a communication system to manage this situation using the steering and speed controllers that were developed in [18].

Under the PATH subproject "Enhanced Coordination and Link Layer Control Algorithms for Improving AHS Capacity," Horowitz et al. [19] presented an efficient lane-change maneuver control system for platoons of vehicles. They remarked upon the fact that the previous PATH lane-change architecture was not efficient at supporting the lane change within platoon operations and presented a new longitudinal controller for lane-change tracking. This longitudinal controller works together with the lateral controllers to optimize the length of the maneuver. Hatipoglu et al. [20] reported the design of an automated lane-change controller. This system requires a twolayer hierarchical architecture. The low-level layer includes two controllers - one for lane keeping and another one for lane changing. The high-level layer gently switches from one lowlevel controller to the other. The system navigates following magnetic markers that are placed in each lane of the road. The lane-change maneuver is conceived as the movement from one lane to the contiguous lane, where navigation is by dead reckoning until the vehicle locates the new lane magnetic sensor sequence. In this instance, we assume that roads are straight for correct system performance.

An exhaustive analysis for calculating the lane-change trajectory is conducted in [21]. It also defines the four situations in which a lane change is safe or unsafe when circulating on freeways. Consider that the overtaking vehicle is vehicle 1 , and vehicle 2 is circulating in the contiguous lane.

Case 1) Vehicle 1 is moving at a lower speed than the other vehicle; vehicle 1 performs a lane change without modifying its speed and pulls in behind vehicle 2 .

Case 2) Vehicle 1 is driving slower than vehicle 2 and makes a lane change, constantly accelerating to pull into the lane in front of vehicle 2.

Case 3) Vehicle 1 is driving faster than vehicle 2 and makes a lane change at a constant speed to pull into the lane in front of vehicle 2 .

Case 4) Vehicle 1 is moving at a higher speed than vehicle 2 and makes a lane change, constantly decelerating to pull in behind vehicle 2 .

Some European projects take a similar approach, where the aim is not to automate the overall maneuver execution but to track maneuver while assisting the driver to get into the right lane and avoid obstacles, as well as advising him or her of any vehicles circulating in a collision trajectory. This is true of the SAFESPOT or PReVENT Integrated Projects (IST-2005026963 and IST-2003-507075), which are deploying driving aids to improve road safety and halve the number of road deaths by 2010 .

In the EU CyberCars-2 Project (IST-2005-028262), the objective is to create a new concept of a cybernetic road transport system, where autonomous vehicles play a central role in urban people mobility. This project proposes a set of cooperative maneuvers by a set of autonomous vehicles. Some of these maneuvers are merging and splitting from a platoon and performing the respective lane changes.

In Japan, important automatic driving system research is being conducted at the National Institute of Advanced Industrial Science and Technology by Prof. Tsugawa's team. Kato et al. [22] presented an automatic driving system that manages longitudinal and lateral controls. This system could manage route tracking maneuvers, as well as stop and go, adaptive cruise control (ACC), and platooning, allowing lane changes for vehicles merging or splitting from the platoon but always on highways with multiple one-way lanes.

From the point of view of complexity, there is a clear difference between a simple lane-change maneuver and an overtaking maneuver. An overtaking maneuver is a sequence of a lanechange maneuver, a path tracking along the new lane, and a return to the original lane; it requires a much greater degree of planning. First, there is the decision whether to initiate an overtaking maneuver. Then, after deciding that the maneuver is possible and necessary, the sequence of partial maneuvers is to be coordinated, so the vehicle returns to the original lane as soon and as safely as possible.

We also have to take into account that a car is an example of a typical system where driving models, sensorial information, objectives, constraints, and control actions are essentially inaccurate. In this paper, we have used fuzzy logic to design lateral controllers. The main reason for using this approach is that a suitable driving process model is essential for automatic steering wheel control. Nevertheless, classical approaches frequently fail to yield appropriate models of complex 
(nonlinear, time-varying, ill-defined) processes-and driving a car certainly falls into this category-whereas fuzzy-logicbased control methods provide an alternative tool for dealing with car and subsystem complexity. In particular, Sugeno and Nishida [23] demonstrated that fuzzy control was capable of handling nonlinear control problems to maneuver a model car using oral instructions.

In this paper, we present an evolution of the simple automatic lane-change system that upgrades autonomous vehicles to use this maneuver to perform complete overtaking operations. We propose a two-level architecture. The low level consists of two fuzzy steering controllers-one for path tracking, which was previously presented in [24], and the other for lane change. Each controller is defined by only two linguistic variables and four fuzzy rules. This configuration reduces the system's complexity without any loss in performance. The aims of the high-level layer of the architecture are to evaluate the necessity and the possibility of overtaking and to switch from one lowlevel controller to the other when appropriate. Speed control is also autonomously managed, keeping the vehicle either at the right speed (cruise control) or at a safe distance from the lead vehicle when overtaking is not possible (ACC) [25]. The main sensorial information is obtained from a high-precision Global Positioning System (GPS) and a wireless communication system.

This paper is organized as follows. Section II presents a brief summary of the project's results to give a better understanding of the overtaking maneuver. Section III describes this maneuver. The software agent in charge of overtaking is described in Section IV, and some experiments are outlined in Section V.

\section{VEHICLE INSTRUMENTATION}

These results are part of the Spanish National Research Council's Autopia Program. The main goal of this program is to develop unmanned vehicles and test fuzzy-logic-based artificial cybernetic drivers. Two Citroën Berlingo vans were automated and instrumented [26] to incorporate an automatic driving system that is installed on an onboard computer. This computer can manage and automatically drive the vans. The main sensors that are used for the navigation and overtaking system are a to-thecentimeter-accurate real-time kinematic differential GPS (RTK DGPS), an onboard speed sensor, a steering angle encoder, and an IEEE 802.11-compliant wireless network, which is used to gather the navigation information from the other vehicles that are involved in the overtaking operation.

\section{A. Route Tracking}

The route tracking system that is installed in our automated vehicles is based on the information supplied by the GPS. It digitally maps the driving zone around which the vehicles circulate like commercial navigators do. As each vehicle carries a GPS receiver, they all know one another's positions and any trajectory error from the reference map defining the route to be followed.

As soon as the system knows the GPS route to be followed, we can calculate the deviations of the vehicle from this route from the GPS positions that are supplied by the onboard receiver. The system can also locate and map all the other vehicles that are circulating around the driving zone. We can then take action, depending on whether these vehicles are in our way.

\section{OVERTAKIng MANEUVER}

An overtaking maneuver is generally used to pass a vehicle that is stationary or driving slower than we are in the same lane. This operation can be performed on two-way roads as well as on freeways, where there is more than one lane in either direction. Although the preconditions that must be met to safely perform the maneuver differ, the performance of the operation is exactly the same: a lane change to the contiguous left lane, trajectory tracking in the left lane until the overtaking vehicle has passed the other vehicle or obstacle in the right lane, and a second lane change to go back to normally circulating in the right lane (Fig. 1).

According to this scheme, each overtaking maneuver includes two lane changes and a path-tracking component. Consequently, the autonomous vehicle's speed and steering need to be managed. Both controls are considered to be partially decoupled. This means that these tasks can be independently executed; however, they share the input information and decision-making layers and work in a coordinated way. The speed control works as an ACC and is described in [25]. It maintains a reference speed for overtaking and obstacle-free circulation and a safety headway when there is a vehicle ahead. We have defined an architecture to support steering control in overtaking maneuvers.

\section{A. Architecture of the Automatic Overtaking System}

When designing an architecture that emulates human driving, we have to look at how humans organize the driving task and what operations they perform.

According to psychologists, human driving can be divided into three activity levels, depending on the attention, resources, and perception that are applied. These are the strategic, tactical, and control levels [27]. The strategic level includes planning and selects, for example, the best route to reach a destination. The tactical level executes complex maneuvers like stopping, overtaking, yielding, etc. Last, the control level performs basic actions to keep the car on the right trajectorymoving the steering wheel and pressing the throttle or brake. These levels are ranked in descending order of complexity. This implies that the higher the complexity becomes, the greater the reasoning that is required, and the less reactive the system is.

A control system based on human behavior, which will support automated operation, has to be built around an architecture paradigm. In our case, we have chosen the Michon [27] model, implemented as a hierarchical architecture, which is capable of supporting automatic driving and can be upgraded to deal with other human-driving scheme maneuvers. Our architecture is, thus, divided into six elements, as shown in Fig. 2.

The strategic layer of the architecture has not been represented in the figure because, in our case, the strategic planning stage is performed by a human user that manually selects the route to be tracked. 


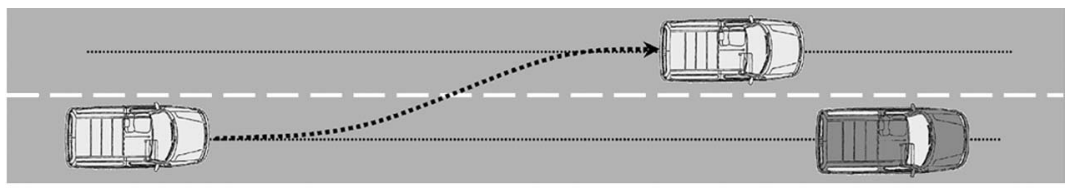

(a)

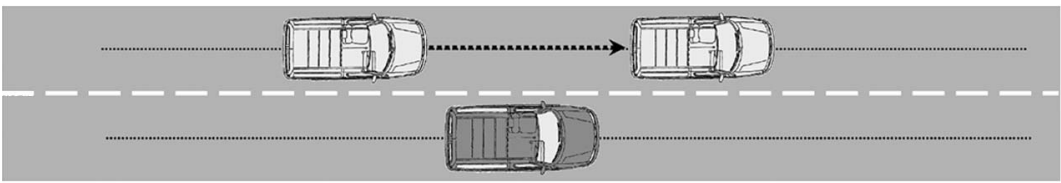

(b)

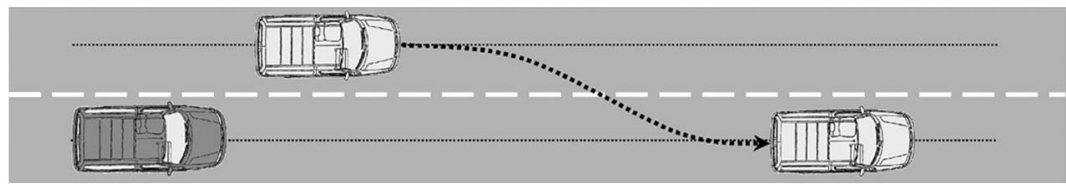

(c)

Fig. 1. Overtaking maneuver phases. (a) First lane change to the contiguous left lane. (b) Circulation in the left lane. (c) Second lane change to the right lane.

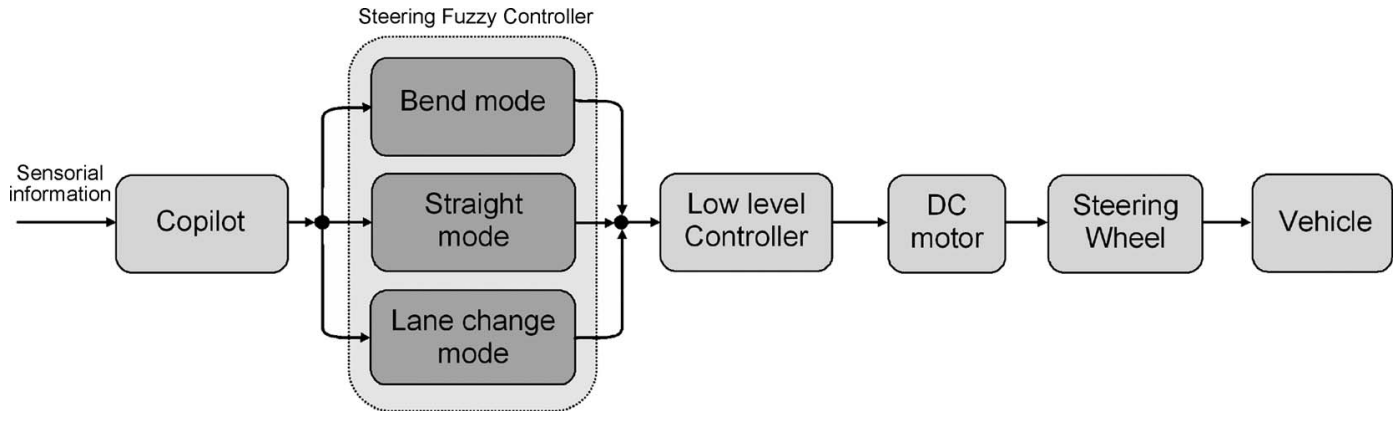

Fig. 2. Hierarchical architecture for steering wheel control in overtaking operations.

The first module is named copilot and emulates the tactical layer of human driving. This is a decision-making module whose mission resembles the job of a rally copilot. It tells the driver when the vehicle is entering a bend or a straight part of the route, when to increase or decrease the target speed, or when it is possible and necessary to overtake, that is, it manages the sequence of operations to be done. Usually, the copilot keeps to the reference route in the right lane. When an overtaking operation starts, it changes the reference route to the left lane and keeps to it until the vehicle it is overtaking has been passed. Then, the reference route returns to the right lane. The copilot checks all the input variables, selects the right driving mode to continue the automatic route, and assures a smooth changeover from one mode to another, that is, it provides for passenger comfort. It can choose between three kinds of steering behavior controllers - bend, straight road, or lane change. These controllers represent the control layer of human driving. These three behaviors are modeled using fuzzy logic. This technique can apply the knowledge of an expert operator (in this instance, a human driver) to control the equipment [23]. Another advantage is that complex mathematical models [28] are not needed to manage the equipment. This is a very useful feature where hard nonlinear systems, like vehicle steering, are concerned. In other words, by applying fuzzy logic to control the steering of a car, we are modeling the driver's, and not the vehicle's, behavior and responses. These fuzzy controllers output the target steering turning to complete the maneuver.

The third architecture module is the low-level controller. Its mission is to receive the target steering wheel angle from the active fuzzy controller and generate the appropriate control signals for the motor to move the steering bar and, hence, the direction in which the vehicle is moving. A PID, which is tuned to manage the dc motor and is attached to the steering column, forms this low-level controller that receives the steering target position from the fuzzy controller and moves the steering wheel until it reaches the target.

The fourth, fifth, and sixth architecture modules consist of the actual dc motor engaged by a gear to the steering bar that turns the wheels and, consequently, the moving vehicle.

This architecture is very similar to that of Hatipoglu et al. [20]. In both architectures, two modules are defined to execute the control layer tasks-lane keeping and lane change-and each behavior is selected from a high-level tactical layer. The difference between these two architectures is basically that, in our case, we add a higher module in charge of strategic planning. However, the functionality of both architectures is identical. On the other hand, the techniques that are used to implement the two architectures are completely different. In our case, the system is based on fuzzy logic controllers and GPS guidance. The Hatipoglu et al. architecture is based on analytical controllers and dead-reckoning systems. 


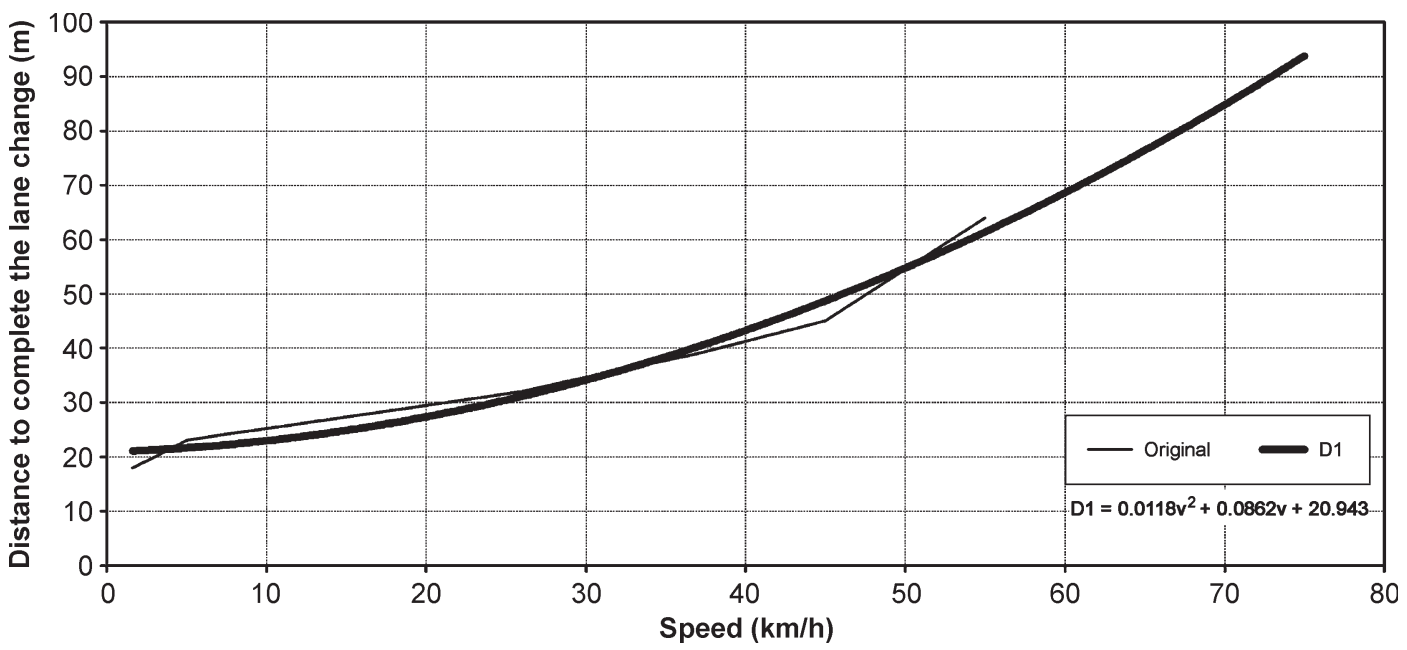

Fig. 3. Experimental relationship between the speed and the lane-change distance.

\section{OVERTAKing CONTROLlER}

As mentioned above, the copilot module manages the overtaking maneuver. It controls its two main aspects: 1) checking whether the conditions are right for overtaking and 2) doing the whole maneuver by accordingly selecting the appropriate fuzzy low-level controllers.

We define a set of conditions to be met by the vehicle environment and the lead vehicle to assure a safe overtaking operation as follows.

1) The overtaking vehicle is moving along a straight road.

2) The overtaken vehicle is moving in the same lane as the overtaking one.

3) The speed of the overtaking vehicle is higher than the speed of the lead vehicle (this means that it has to be overtaken).

4) The left lane is free.

5) The lane is long enough for the overtaking maneuver to be completed at the current speed.

The first condition is verified by checking the selected driving mode. The second depends on the GPS position of the two vehicles, and the fourth depends on the fact that no GPS position of any other vehicle is detected in the left lane. If a vehicle is detected, we need to know whether it can affect the maneuver. The last condition is harder to calculate; the system must make sure that there is enough road space for the permitted overtaking maneuver to be completed. This estimate is described in Section IV-A.

\section{A. Overtaking}

If all these constraints are met, then the overtaking maneuver can start. We define the sequence of operations that must be executed to undertake the maneuver.

1) Compute the time when the first lane change starts.

2) Perform the first lane change to the left lane.

3) Circulate in the left lane until the lead vehicle has been passed.

4) Return to the right lane.

5) Continue to normally circulate.
TABLE I

Lane Change Distance and Car SpeEd

\begin{tabular}{cc}
\hline \hline $\mathrm{V}_{1}^{\prime}(\mathrm{km} / \mathrm{h})$ & $\mathrm{D}_{1}(\mathrm{~m})$ \\
\hline 1.6 & 18 \\
3 & 20 \\
5 & 23 \\
7 & 24 \\
9.6 & 25 \\
26 & 32 \\
29 & 34 \\
37 & 39 \\
45 & 45 \\
55 & 64 \\
\hline \hline
\end{tabular}

Initially, the car's reference route is the right lane of the road, and the straight-path fuzzy controller manages the steering wheel.

The first operation determines when the overtaking vehicle has to start to make the first lane change into the left lane of the road. The condition for this operation is that the lane change must have finished by the time the front part of the overtaking vehicle is at the same $X$ coordinate as the overtaken one's rear (Fig. 3). Let us not forget that vehicle 1 follows vehicle 2 using the ACC. Then, it accelerates to its target speed while it changes lanes. Its acceleration is nonlinear and depends on the initial speed and fuzzy rules that control the speed. Furthermore, the vehicle might reach the target speed before the lane change has been completed, and then, it will stop accelerating. Next, the overtaking vehicle will circulate for a time in the right lane, approaching the overtaken vehicle until its front part reaches the rear part of vehicle 2. For safety reasons, we define the distance $D$ as the separation between the overtaking and overtaken vehicles at which the overtaking maneuver must start. This complies with the condition of minimizing the time that the overtaking vehicle circulates in the left lane. This distance will be a function of the speeds of both vehicles involved, i.e., $D=f\left(v_{1}, v_{1}^{\prime}, v_{2}\right)$, where $v_{1}$ is the speed of the overtaking vehicle at the beginning of the maneuver $\left(t_{1}\right)$, $v_{1}^{\prime}$ is the target speed of this vehicle, and $v_{2}$ is the speed of the overtaken vehicle, which is assumed to be constant. For our purposes, we consider $v_{1}^{\prime}$ only because it is the maximum speed that the vehicle will reach, and it is impossible to estimate 


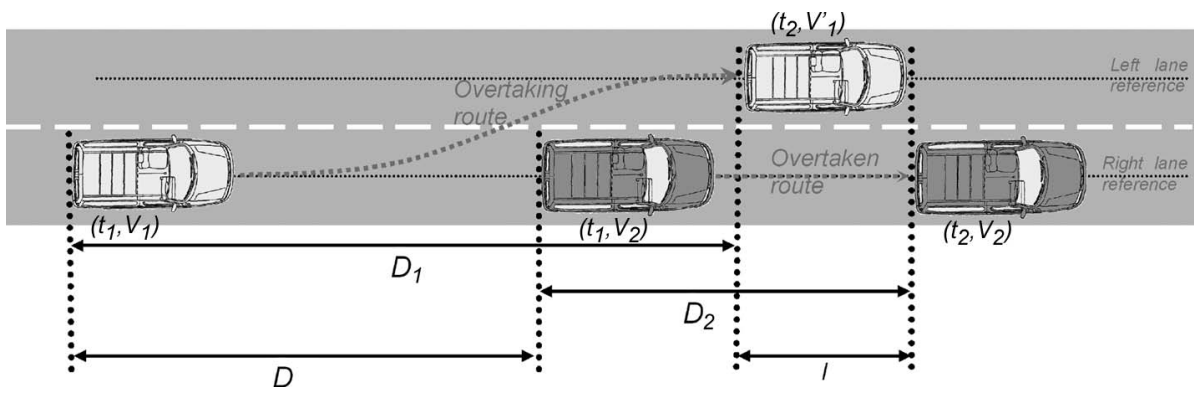

Fig. 4. Overtaking maneuver variables for starting distance calculation.

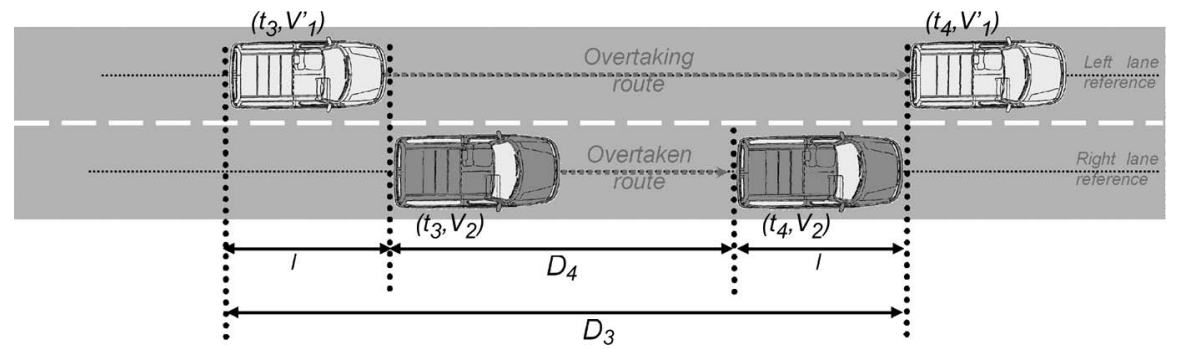

Fig. 5. Left-lane track for passing the overtaken vehicle.

the vehicle's acceleration during the maneuver. $v_{1}^{\prime}$, therefore, will be the target speed that is selected by the cruise control as the maximum speed for the road. We will also define $D_{1}$ as the longitudinal distance that it takes the vehicle to change to the contiguous lane and $D_{2}$ as the distance the overtaken vehicle moves during this lane change. It is not easy to determine $D_{1}$ a priori using analytical means. Therefore, we have used a classical engineering method; we have searched for a least squares solution minimizing a series of points $\left(D_{1}, v_{1}^{\prime}\right)$ taken at different initial speeds $v_{1} . D_{1}$ will, thus, be nontime dependent because its value is empirically calculated by running a number of automatic lane-change experiments using our controllers and defining the outline of the function that estimates the distance that it takes to change a lane. Table I shows the results of the lane-change experiments for several lane changes at different speeds $\left(v_{1}^{\prime}\right)$.

In this table, each different value of the distance has been obtained by performing five experiments at the corresponding speed and rounding the result to the nearest integer. The experimental data collection was conducted by performing an automatic lane change at the corresponding speed while registering the route as tracked by centimeter-accuracy GPS coordinates.

These data can be used to obtain the equation that states the relationship between the speed of a vehicle and the distance that it takes to complete a lane change. Fig. 3 shows these data as well as the adjusted second-degree polynomial for (1).

Now, we can calculate the distance $D$ as follows to begin overtaking:

$$
\begin{aligned}
D & =D_{1}+l-D_{2} \\
D_{1} & =0.0118 v_{1}^{\prime 2}+0.0862 v_{1}^{\prime}+20.943 \\
D_{2} & =v_{2} \cdot \Delta t=v_{2} \cdot\left(t_{2}-t_{1}\right) \\
\Delta t & =\frac{D_{1}}{v_{1}^{\prime}} \\
D & =l+D_{1}\left(1-\frac{v_{2}}{v_{1}^{\prime}}\right)
\end{aligned} D_{2}=D_{1} \frac{v_{2}}{v_{1}^{\prime}}
$$

where $l$ is the length of the overtaking vehicle $(4 \mathrm{~m}$ for the Citroën Berlingo van).

Formula 2 can also be used to calculate the fifth constraint on overtaking; make sure that the lane is long enough for the overtaking maneuver to be completed at the current speed. To check whether this condition is met, we have to estimate the distance that it takes to complete the maneuver (Fig. 4). This distance is an addition of the distances that it takes to execute the three parts of the maneuver: $D_{1}$ for the first lane change and the distance from when the overtaking vehicle finishes the first lane change until it completely passes the overtaken car $\left(D_{3}\right.$; Fig. 5) for left-lane tracking.

With this information, we can estimate the distance the vehicle is to travel, i.e.,

$$
\begin{aligned}
& D_{3}=2 l+D_{4} \\
& \left.\begin{array}{l}
D_{3}=v_{1} \cdot t \\
D_{4}=v_{2} \cdot t
\end{array}\right\} D_{4}=v_{2} \frac{D_{3}}{v_{1}} \\
& D_{3}=2 l+v_{2} \frac{D_{3}}{v_{1}} \\
& D_{3}=\frac{2 l \cdot v_{1}}{v_{1}-v_{2}} \text {. }
\end{aligned}
$$

Last, we consider that the time taken for the second lane change is the same as that for the first lane change, i.e., $D_{1}$. Then, the estimated distance for overtaking is given by

$$
D_{\text {overtaking }}=2 D_{1}+D_{3} \text {. }
$$

The second step in overtaking is to perform the first lane change to the left lane. This operation is done by switching to the lane-change fuzzy steering controller and selecting the left lane of the road as the reference route. No trajectory definition is necessary to perform this lane change. Once the lane-change operation is complete, the system keeps the left lane as the reference route but selects the straight-path-tracking fuzzy controller until it passes the overtaken vehicle. When 
TABLE II

SteEring Control FuZzy Rule BASE

\begin{tabular}{ll}
\hline \hline$R 1$ & IF lateral_error right THEN steering left \\
$R 2$ & IF lateral_error left THEN steering right \\
$R 3$ & IF angular_error right THEN steering left \\
$R 4$ & IF angular_error left THEN steering right \\
\hline
\end{tabular}

the overtaken vehicle has been passed, the overtaking vehicle returns to the right lane. To do this latter operation, we select the lane-change fuzzy controller, and the right lane is defined as the reference route. Last, as soon as the vehicle is centered in the right lane, the straight-path-tracking fuzzy controller is selected, and the vehicle continues to circulate normally.

\section{B. Fuzzy Lateral Controller}

Fuzzy controllers do the computation for the steering control. From a formal point of view, a fuzzy controller consists of a rule base containing the experts' procedural knowledge and a variable base containing the different linguistic values that they consider.

Fuzzy rule base: The human driver's procedural knowledge is represented by a fuzzy rule base. This rule base contains the necessary information on how drivers execute their actions to keep the vehicle to the target route. In this instance, the fuzzy rule base is common to all driving modes because the driver's main objective is to keep the vehicle to the route, i.e., to minimize the lateral as well as angular errors.

Therefore, only four rules are necessary to keep the vehicle in the lane or to perform a lane change. They are shown in Table II, where the words in italics are the fuzzy input and output variables, and the words in bold are their associated linguistic values.

These rules stand for human reasoning: If the vehicle is moving out of the lane to the left, then turn the steering wheel to the right to offset the deviation. The same thing applies if the deviation is to the right.

Fuzzy variable base: We define two linguistic variables named lateral_error and angular_error. These two variables have two associated linguistic values named (left, right), each with their respective membership function. The shape of the membership functions depends on how and how much we want these variables to affect the control. This feature is illustrated in the following sections, where we differentiate the driver's behavior in straight path tracking and lane change by differently modeling the associated membership functions. Last, we define a fuzzy output variable, named steering, whose linguistic labels are (left, right).

From a functional point of view, the fuzzy reasoning process can be divided into three stages-fuzzification, inference engine, and defuzzification.

Fuzzification: In this step, current crisp input values are transformed into linguistic or fuzzy values that can be interpreted by the fuzzy compiler. This transformation computes a degree of truth for each one of the input fuzzy variable values, depending on the shape of their associated membership functions. This represents human drivers' subjective knowledge.

In our case, we consider two input variables for lateral control-lateral and angular errors from the reference route.
These are the distance of the front of the vehicle to the GPS reference route and the angle between the vehicle's direction vector and this route, respectively.

Inference engine: The inference engine propagates the matching of the conditions to the conclusions, generating the contribution of each rule to the control action. In our case, Mamdani's [29] inference method (min-min-max) is used to solve the fuzzy implication.

Defuzzification: Defuzzification is the transformation of the output fuzzy values that are generated by applying the inference method into crisp values that can be used to output control intentions. In this instance, we use the center of area $(\mathrm{CoA})$ method, i.e.,

$$
\begin{aligned}
y_{\mathrm{CoA}} & =\frac{\int B \cdot y d y}{\int B d y} \\
B & =\bigcup_{i} \omega_{i} B_{i}
\end{aligned}
$$

where $\omega_{i}$ represents the membership degree resulting from the inference of the $i$ th rule, and $B_{i}$ is the membership function for the different values of the output variable of the $i$ th rule. This method is very commonly used in control applications [30]-[34].

We have defined the output fuzzy variable membership function shapes using Sugeno's singletons [35], [36], which use monotonic functions. In this instance, a modified CoA equation is applied, i.e.,

$$
y_{\mathrm{CoA}}^{\prime}=\frac{\sum_{i} \omega_{i} B_{i}}{\sum_{i} \omega_{i}} .
$$

Therefore, when speaking of the lateral control, $y_{\mathrm{CoA}}^{\prime}$ would indicate the target turning angle for the steering wheel. This value is normalized and defined in the $[-1,1]$ interval and needs to be multiplied to obtain $540^{\circ}$, which is the maximum the steering wheel will turn.

Thus, the rule base is the same for both controllers; however, the definition of the input membership functions implements the behavioral differences. Next, we show this definition and explain the differences supporting lane keeping and lanechange behavior.

As already mentioned, the human drivers' procedural knowledge (the fuzzy rules) is the same for both driving modes. The difference between straight-lane and lane-change driving lies in the quantification of trajectory errors, which humans unconsciously do. This is the definition of the membership functions for the input and output variables. These membership functions have been tuned to mimic human behavior in the respective situations.

1) Straight-Road Fuzzy Controller: Fig. 6(a) and (b) shows the definition of these functions for the lateral and angular errors, each with three linguistic labels (right, center, and left). They depend on the sign and the magnitude of the trajectory error.

Circulation on straight roads is usually very fast, and consequently, the permitted trajectory errors are very small. Accordingly, the shape of the membership functions has been 


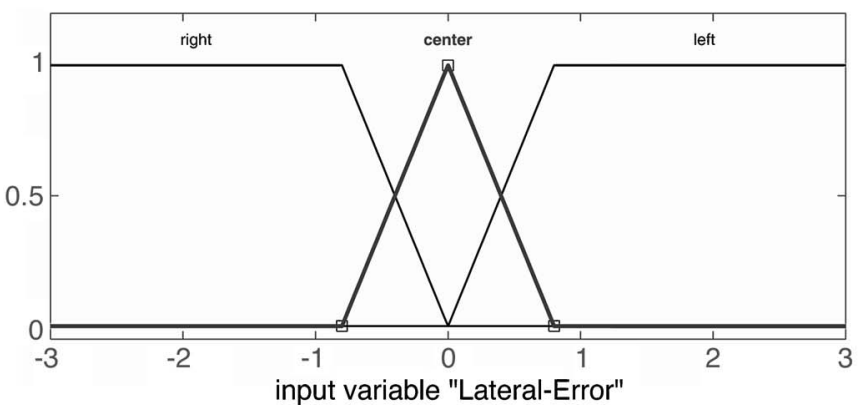

(a)

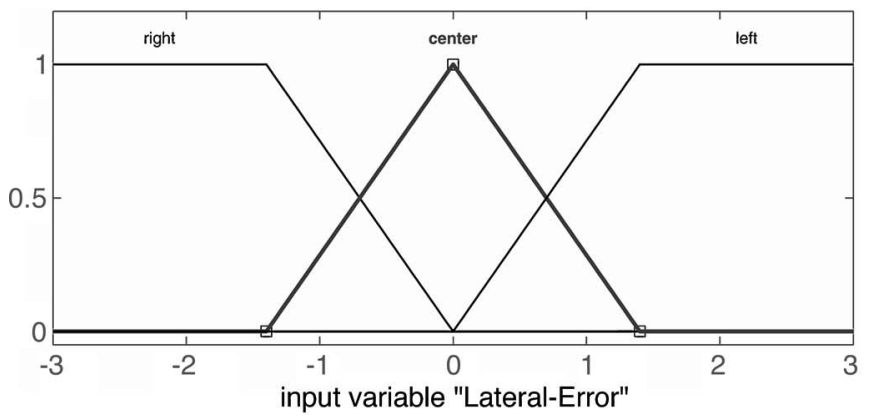

(c)

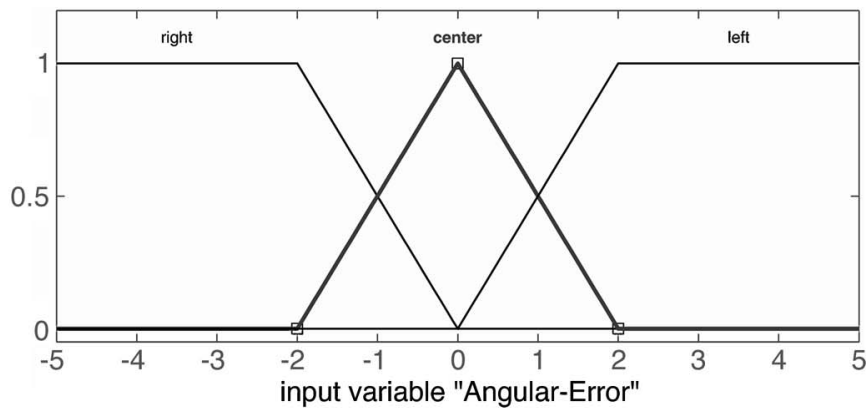

(b)

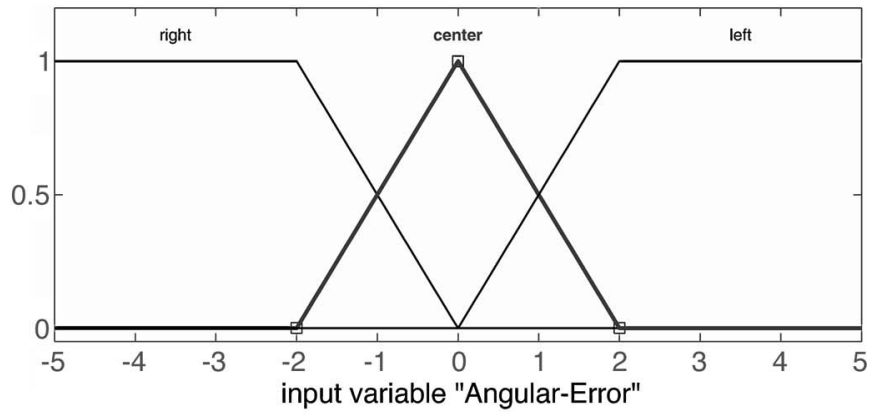

(d)

Fig. 6. Membership function definition for the input fuzzy variables. (a) Straight-path-tracking error. (b) Straight-path-tracking angular error. (c) Lane-change lateral error. (d) Lane-change angular error.

defined as very sharp to make the system very reactive and stop the vehicle that is moving out of the lane.

The lateral error fuzzy variable contains three membership function definitions for each of its three associated linguistic labels. The membership function for the center linguistic label has been defined in the $[-0.8,+0.8] \mathrm{m}$ interval; this means that the maximum membership degree is when the crisp value of the lateral error is $0 \mathrm{~m}$, and this membership degree decreases following a linear function to the limits of this interval, where the membership degree is null. The aim of this definition is to qualify how centered the vehicle's trajectory is in the road.

Similarly, the left linguistic label has a related membership function whose aim is to define how far deviated to the left the vehicle is from its target trajectory. This membership function is defined in the interval $[0, \infty]$, where the membership degree is minimum (null) at $0 \mathrm{~m}$, the degree between 0 and $0.8 \mathrm{~m}$ increases following a linear function, and the maximum membership degree is maintained in the remaining interval.

The right linguistic label has an associated linguistic function defined in the $[-\infty, 0]$ interval, whose objective is to define how far deviated to the right the vehicle's trajectory is.

Note that these membership functions have been defined as symmetric because we want the system response that is centering the vehicle when it is deviated to the left to be the same as when it centers the vehicle that is deviated to the right. The symmetry does not necessarily apply in every case.

All three linguistic labels are perfectly determined when the position of the $0.8-\mathrm{m}$ vertex is known. The value of the membership function vertex was experimentally fine tuned by studying the effect of this value on the system response.

All the points that have been made for the lateral error also apply to the angular error. The linguistic label center has a maximum value when the vehicle is parallel to the target trajectory $\left(0^{\circ}\right)$ and linearly decreases to 0 when the angle is $2^{\circ}$. The right and left linguistic values also peak when the absolute angle is greater than $2^{\circ}$ and linearly decrease to 0 when the vehicle is parallel to the reference trajectory. As with the linguistic values of the lateral error fuzzy variable, the $2^{\circ}$ value has been experimentally determined to be the one yielding the best response.

These linguistic labels imply that when the deviation is greater than $\pm 0.8 \mathrm{~m}$, rules $\mathrm{R} 1$ or $\mathrm{R} 2$ will be the only contribution to the steering movement.

The angular error membership function has the same effect on the steering output variable. The contribution of rules R3 and R4 is maximum when the crisp error is higher than $\pm 2^{\circ}$. This falls when this variable value decreases from $\pm 2^{\circ}$ to $0^{\circ}$.

These definitions assure that the deviation of the vehicle's trajectory is always less than $\pm 0.8 \mathrm{~m}$ and $\pm 2^{\circ}$ because the control system will act before the errors reach this level to prevent them from occurring.

The output fuzzy variable, named steering, also has two linguistic labels (left and right), whose membership functions are defined as Sugeno's singletons. The normalized monotonic functions are defined at -0.025 and 0.025 , respectively. These values have also been experimentally determined, and their meaning is clear-to limit the total output to $2.5 \%$ of the maximum. The reason for this is that the steering wheel must move very carefully when the vehicle moves along a straight lane. Of course, this does not apply to bend driving, where the steering wheel output is unlimited [24].

2) Lane-Change Fuzzy Controller: The lane-change fuzzy controller is designed to use the same rule set as the pathtracking controller, albeit for a different mission. In this 


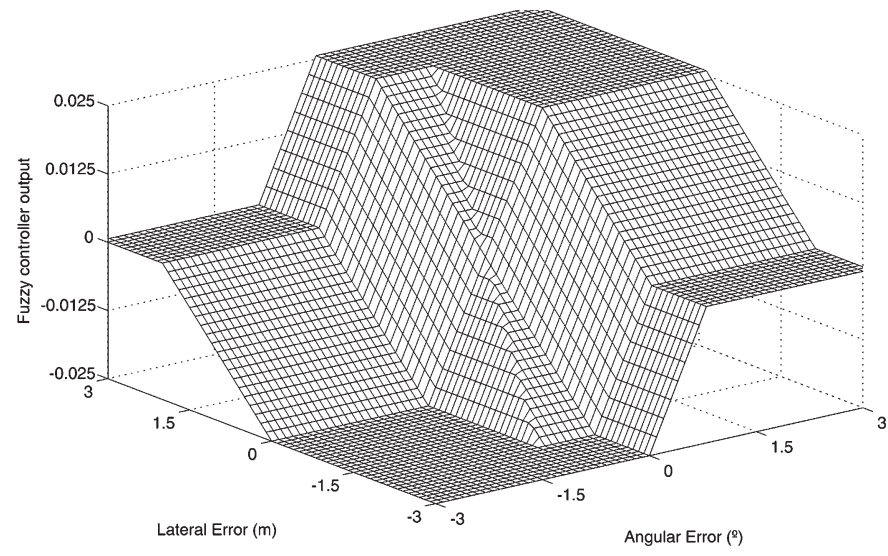

(a)

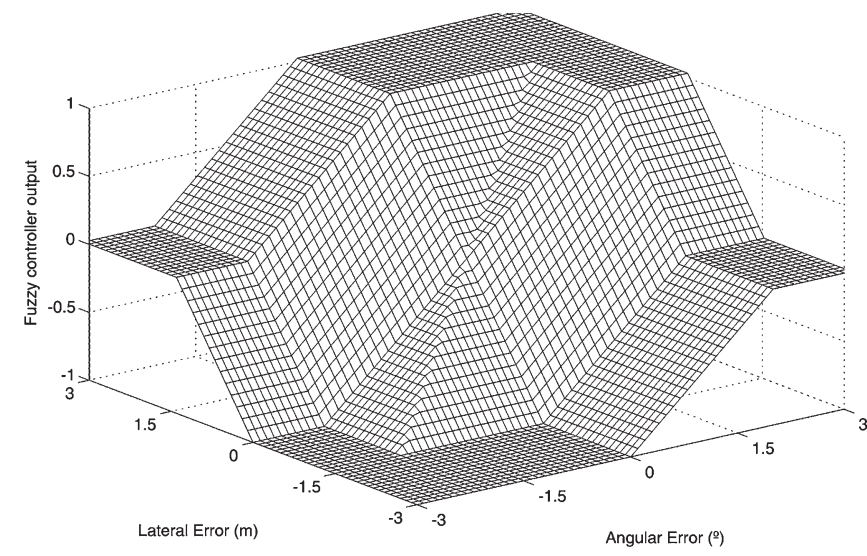

(b)

Fig. 7. Control surfaces for the fuzzy controllers. (a) Straight-road controller. (b) Lane-change controller.

instance, the mission is to control the vehicle when its reference trajectory changes from a reference lane in the right lane of the road, which is defined as a sequence of GPS coordinates, to an alternative trajectory that is located in the contiguous left lane, or vice versa. The lane-change controller does not generate a trajectory for the change of lane. Rather, it makes the vehicle smoothly adjust its route so that it naturally adapts to a new reference trajectory in the new lane.

Fig. 6(c) and (d) shows the definition of the membership functions for the lane-change controller. The vertex in the angular error linguistic label definition is the same as that for path tracking. The reason is that the maximum angular error during the lane change should be kept under $\pm 2^{\circ}$ to assure that the maneuver is smoothly executed. On the other hand, the lateral error membership function definition vertex has been defined at $\pm 1.5 \mathrm{~m}$. This distance is half the width of a lane. This means that, at the beginning of the lane change (when the vehicle is circulating in the right lane), the reference is changed to the contiguous lane, and then, the lateral error becomes about $3 \mathrm{~m}$, considering that the lane is about $3 \mathrm{~m}$ wide. Therefore, the maximum membership degree for the contribution of rules R1 or R2 (whichever is applicable) is when the vehicle is located between the starting position and the lane's dividing line (as the lateral error changes from 3 to $1.5 \mathrm{~m}$ ). As the lane change proceeds, the lateral error decreases to less than $1.5 \mathrm{~m}$, and the contribution of the rule drops in the final control signal because the membership degree also falls from 1 at $1.5 \mathrm{~m}$ to 0 at $0 \mathrm{~m}$.

With this configuration, at the beginning of the lane change, the lateral error induces the vehicle to get into the contiguous lane as quickly as possible, whereas when it reaches the center of the road, the angular error makes the vehicle smoothly track the new lane.

In this instance, the output variable steering also has two linguistic labels, i.e., left and right, whose associated singletons are defined as normalized monotonic functions at -1 and 1 , respectively. This means that, contrary to what was said at the end of the preceding section, there is no limit on steering wheel movement. However, we have determined that, for safety reasons, the steering movement of a circulating vehicle must be speed dependent, and a $2.5 \%$ limit has to be set on the maximum output when the vehicle is circulating in a straight lane. Yet, the limitations that are forced on the output to make straight-lane driving easy would prevent the vehicle from smoothly changing lanes. Thus, we have determined a function where $v_{r}$ represents the real speed of the vehicle, and $v_{t}$ represents the target speed that is selected by the control system. The value that this function yields will multiply the crisp output of the fuzzy controller to obtain the final output. The criterion used to obtain this function is that the coefficient is 0.025 for high speeds [where a high speed indicates that the mean speed $\left(v_{r}+v_{t}\right) / 2$ is greater than $66 \mathrm{~km} / \mathrm{h}$ ], linearly varying up to a value 0.147 for low speeds, which is a value that has been experimentally determined.

This function is shown in the following:

$$
\begin{aligned}
f\left(v_{r}, v_{t}\right)= & -0.00185 \cdot \frac{1}{2}\left(v_{r}+v_{t}\right)+0.147 \\
& \Leftrightarrow \frac{1}{2}\left(v_{r}+v_{t}\right) \in[0,66] \mathrm{km} / \mathrm{h} \\
f\left(v_{r}, v_{t}\right)= & 0.025 \Leftrightarrow \frac{1}{2}\left(v_{r}+v_{t}\right)>66 \mathrm{~km} / \mathrm{h} .
\end{aligned}
$$

Fig. 7 shows the response surfaces that represent the input/ output mapping according to the fuzzy rules. The surfaces are smooth, which means that the rules are reasonable.

\section{EXPERIMENTS}

After we had defined the automatic overtaking system, we ran some field tests to demonstrate that it was correctly configured. We used two vehicles. The first, i.e., the overtaking vehicle, was unmanned, and the second, i.e., the overtaken vehicle, was manually driven. Although both vehicles could circulate in the automatic mode, we opted to test automatic driving against human driving because human behavior is more unpredictable than automatic actions. This should give an idea of the flexibility of the driving system developed. Each experiment is explained in a figure with two graphs. The top graph represents the speeds of both vehicles during the automatic overtaking test in kilometers per hour. The second graph represents the straight road where the experiments were performed. Two horizontal black lines define the road borders, and one central black dotted line is the separation between the two lanes. This graph represents the trajectory followed by both 

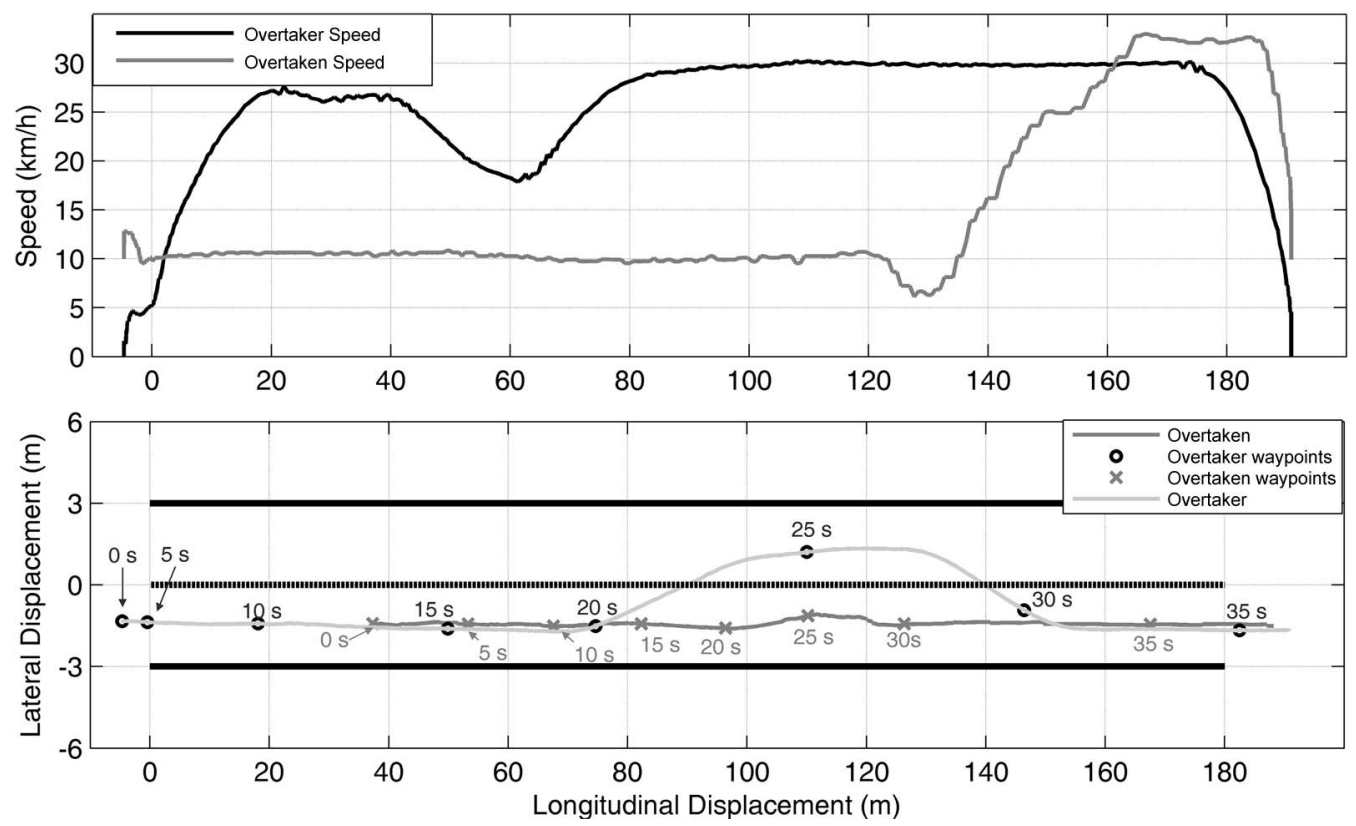

Fig. 8. Automatic overtaking experiment.

vehicles during the experiment, where the position of the cars at the same time instant is represented by waypoints, which are taken at 5-s intervals.

\section{A. Automatic Overtaking of a Circulating Vehicle}

At the beginning of the first experiment, shown in Fig. 8, the overtaking vehicle is stationary, and the overtaken vehicle is circulating at a speed of about $10 \mathrm{~km} / \mathrm{h}$. Both vehicles are $50 \mathrm{~m}$ apart in the same lane. The target speed for the overtaking and overtaken vehicles is 30 and about $10 \mathrm{~km} / \mathrm{h}$, respectively.

Since the overtaking vehicle is moving at a higher speed than the overtaken one, it closes in on the overtaken car. This activates the ACC, which reduces the speed to maintain a safety headway and permit an emergency stop if necessary. This continues until the distance between them decreases to $D$ [see (1); in this case, $D \sim 70 \mathrm{~m}]$ at $18.2 \mathrm{~s}$ from the start of the experiment. At this point, the tracking controller switches from the ACC straight-lane tracking controller to the lane-change controller, and the left lane is selected as the reference route. The lane-change operation is, therefore, smoothly executed, as shown, with no overshooting of the lateral or angular errors. Last, the lane-change maneuver finishes when the absolute value of the lateral error is less than $0.7 \mathrm{~m}$, and the absolute value of the angular error is less than $5.2^{\circ}$. This happened $24 \mathrm{~s}$ from the start of the experiment, when the distance between the front of the overtaking vehicles and the rear of the overtaken vehicles is $3 \mathrm{~m}$. This means that the design requirements of the overtaking algorithms are satisfied, and the first lane-change maneuver is completed, minimizing the left-lane occupation time. The time taken to complete this first part of the maneuver is $5.2 \mathrm{~s}$.

After the first lane change has been completed, the driving controller switches back to straight-lane tracking using, in this instance, the left lane as the reference route. Owing to the difference in speeds, it takes the overtaking vehicle $4.1 \mathrm{~s}$ to pass the other vehicle. When the overtaking vehicle has completely passed the lead vehicle, the lane-change controller is activated again, and the reference route is modified from the left lane to the right lane.

As shown in Fig. 8, this second lane change is correctly performed, and the return to the new reference lane is executed without overshooting the trajectory. When the overtaking vehicle is centered in the right lane and the absolute value of the lateral and angular errors is less than $0.7 \mathrm{~m}$ and $5.2^{\circ}$, respectively, the straight-path-tracking controller is loaded again, and the overtaken vehicle continues along its normal route. It only takes $3.1 \mathrm{~s}$ to complete this operation. The difference in duration between the first and second lane changes was due to the fact that the vehicle was moving at different speeds each time.

Fig. 8 also illustrates that the switches between the straightand lane-change controllers are smooth because there is no oscillation in the four controller changes.

The evolution of the overtaking vehicle's speed is also noteworthy. Fig. 8 shows that this vehicle's speed decreases by more than $10 \mathrm{~km} / \mathrm{h}$ from the reference speed about $40 \mathrm{~m}$ from the start. The reason for this reduction in speed is that, until the overtaking conditions are met, the overtaking vehicle's speed is adapted to keep a safe distance from the vehicle in front using the system described in [25]. Once the overtaking conditions are met, the speed is only limited by the reference speed, and the vehicle reaccelerates to perform the maneuver. This speed increase is also correctly dealt with by the driving system that adapts the distance $D$ to the reference speed. The overtaken vehicle also behaves strangely. It reduces its speed when the overtaking vehicle passes, but it does not give way to the second vehicle. Do not forget that it is manually driven, and this just reflects the driver's unpredictability. The driver of the vehicle admits to driving his own vehicle this way. In any case, the computations do not put the bodies of any of the vehicles at risk. 

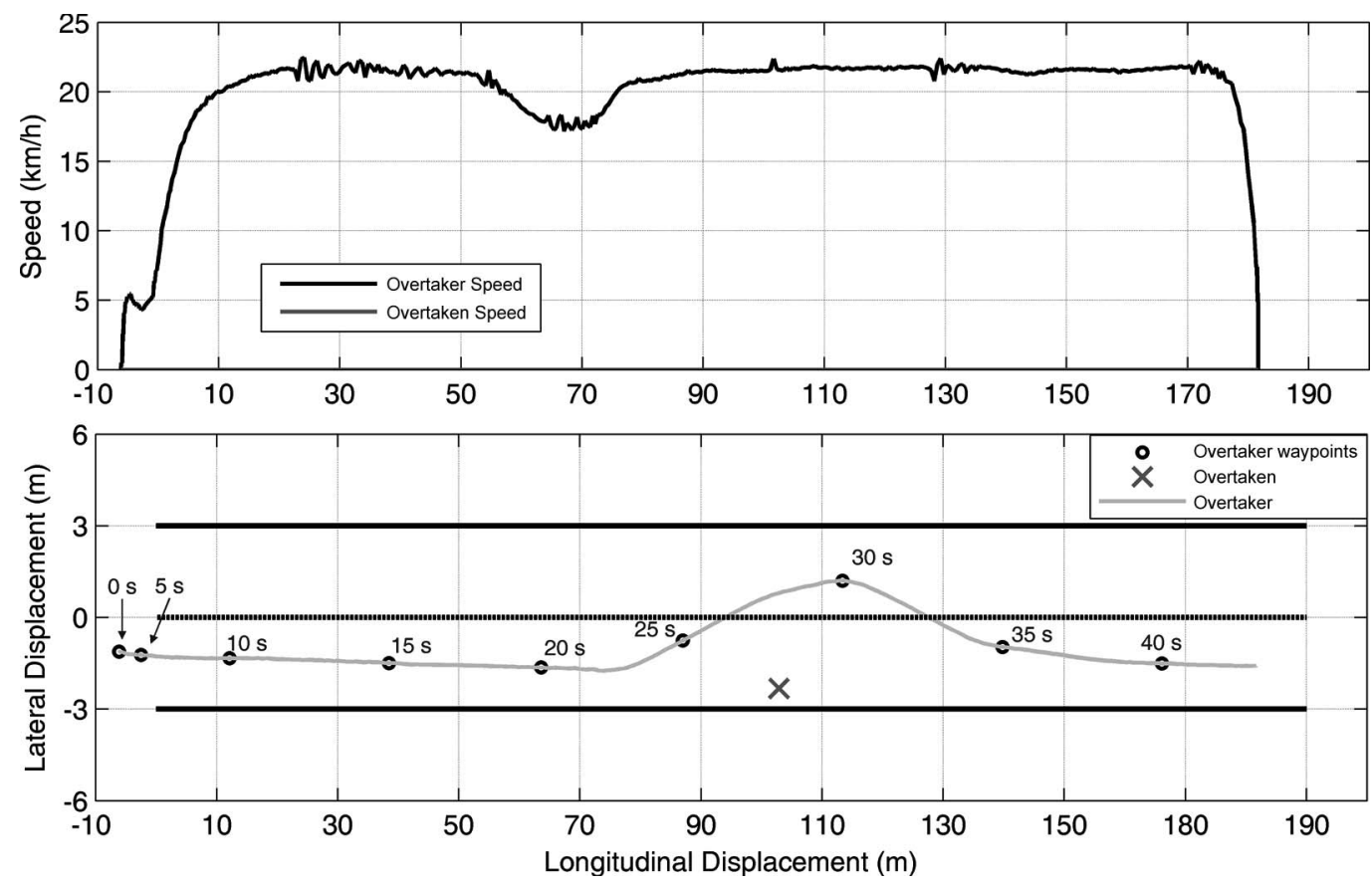

Fig. 9. Overtaking a stationary car.
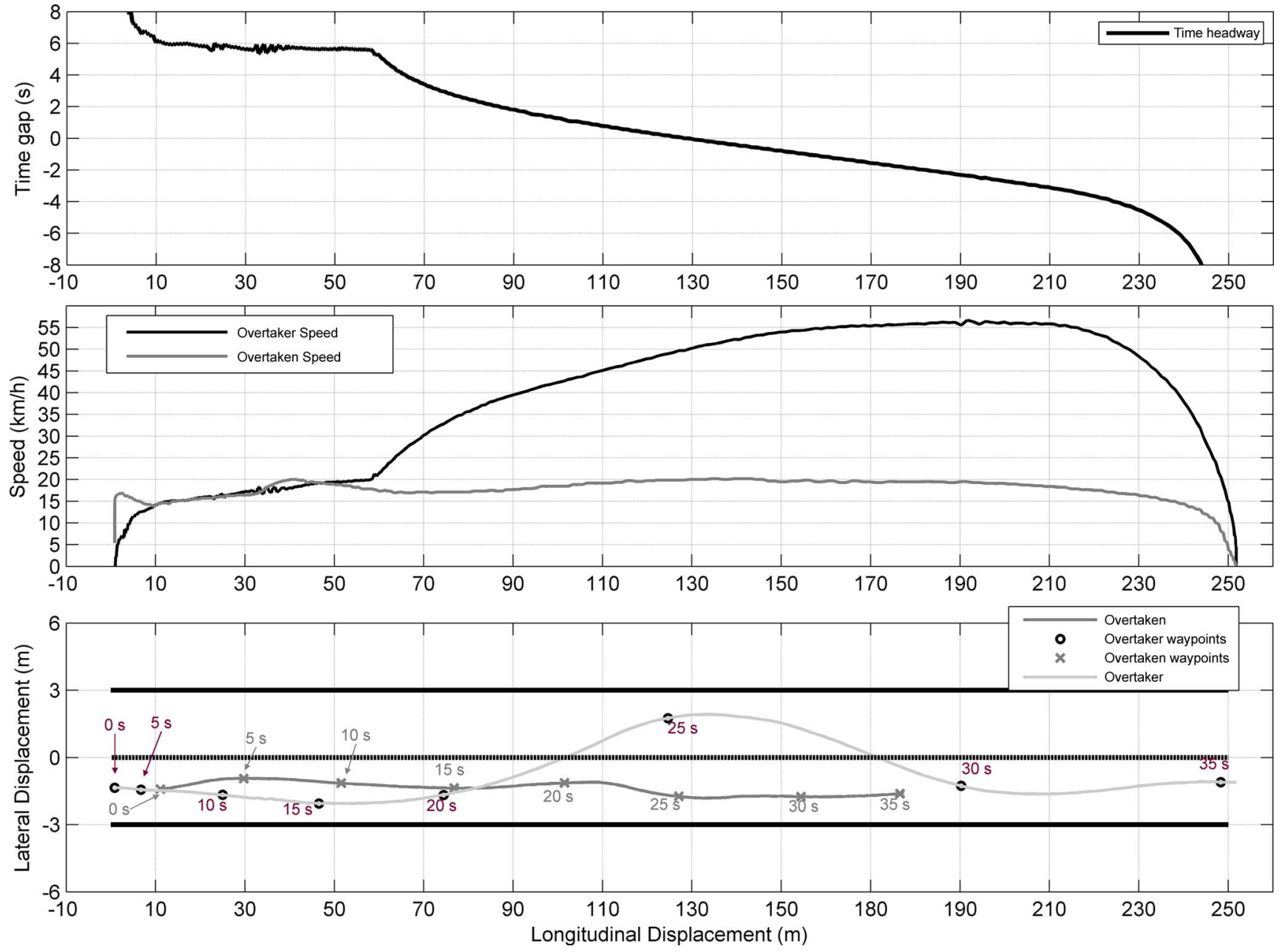

Fig. 10. Overtaking with the ACC. 


\section{B. Automatic Overtaking of a Vehicle Stopped in the Road}

The second experiment, which is shown in Fig. 9, consists of automatically overtaking a stationary vehicle. In this instance, the circulation speed of the overtaking vehicle is set at $21 \mathrm{~km} / \mathrm{h}$ to show the system performance at a different initial setting. As we can see, the speed is maintained all the time, except when the stationary vehicle is detected, and the ACC attempts to keep a safe distance until the overtaking distance is reached. No overshooting appears in the trajectory either when changing to the left lane or returning to the original lane. In this instance, the circulation in the left lane is minimal because the obstacle is a 4-m-long stationary car. Comparing these two graphs, we find that the lane-change distance is about $\sim 46 \mathrm{~m}$ (from meter 64 to meter 110) in the former and $\sim 30 \mathrm{~m}$ (from meter 73 to meter 103) in the latter. This is perfectly coherent with the different vehicle speeds. The faster the vehicle goes, the greater the lanechange distance is because, according to (8), steering wheel movement is more limited.

\section{Combination of the ACC With Overtaking}

The third experiment represents a more complex scenario, where the ACC is combined with the overtaking maneuver (Fig. 10). In this instance, a third graph has been added to show the ACC performance where the safety time headway is preset at a target of $6 \mathrm{~s}$. In this instance, the target speed of the overtaking vehicle is set at $55 \mathrm{~km} / \mathrm{h}$, and the overtaken vehicle circulates about $15 \mathrm{~km} / \mathrm{h}$. Overtaking is not allowed at the beginning of the experiment, and the control system selected keeps a safety headway from the preceding vehicle to avoid a crash. During the first $60 \mathrm{~m}$ of the experiment, the ACC system reduces the overtaking speed and maintains a correct safety headway of 6 s. At meter 60, the overtaking prohibition disappears, and the overtaking vehicle increases its speed to $55 \mathrm{~km} / \mathrm{h}$ and approaches the overtaken vehicle until it reaches the distance at which the overtaking should start. Then, the first lane change proceeds correctly, following the desired smoothness and safety parameters, using the same controllers as in the previous experiments but at a higher speed. Fig. 10 shows that, at the very instant that the first lane change finishes, the overtaking vehicle reaches the same $X$ coordinate as the overtaken vehicle, and the time gap between both vehicles is 0 .

After the first lane change has finished, the straight-lane controller is activated until the overtaking maneuver has finished, and then, a second lane change is executed to return to the original right lane. Fig. 10 also shows that the controller change is smooth, as indicated by the absence of oscillations in the overtaking vehicle's route when the driving mode changes.

In this experiment, the distance traveled by the overtaking vehicle during the first lane change is also consistent with the controller design because the higher the speed, the lower the permitted maximum steering turning. For a target speed of $55 \mathrm{~km} / \mathrm{h}$, the lane-change distance traveled is about $60 \mathrm{~m}$.

\section{CONCLUSION}

In this paper, we have presented an automatic driving system that is capable of performing automatic driving on straight roads and overtaking maneuvers when a slower vehicle appears in the unmanned vehicle's way, and a set of safety conditions is satisfied. This system implements a fuzzy-control-based automatic lane-change system. No specific reference trajectory needs to be defined for this maneuver, as the lane-change system is able to perform this maneuver by just specifying the contiguous reference lane GPS mapping. The navigational information that is needed to do the overtaking operation is supplied by an RTK DGPS governing the navigation of each vehicle and the data gathered from a wireless communication system that supplies the GPS coordinates of the overtaken vehicle in real time. From the information on the GPS position of both vehicles and the GPS mapping of the reference route, the driving system can perform human-like overtaking maneuvers.

\section{ACKNOWLEDGMENT}

The authors would like to thank the Ministerio de Fomento and Ministerio de Educación. They would also like to especially thank Citroën España S.A.-without its collaboration, this paper would not have been written.

\section{REFERENCES}

[1] G. Hegeman, "Overtaking frequency and advanced driver assistance systems," in Proc. IEEE Intell. Vehicles Symp., Parma, Italy: Univ. Parma, 2004, pp. 145-149.

[2] S. Tsugawa, T. Yatabe, T. Hirose, and S. Matsumoto, "An automobile with artificial intelligence," in Proc. 6th IJCAI, Tokyo, Japan, 1979, pp. 893-895.

[3] A. Broggi, M. Bertozzi, A. Fascioli, and G. Conte, Automatic Vehicle Guidance: The Experience of the ARGO Autonomous Vehicle. Singapore: World Scientific, 1999.

[4] A. Broggi, M. Bertozzi, A. Fascioli, C. G. Lo Bianco, and A. Piazzi, "The ARGO autonomous vehicle's vision and control systems," Int. J. Intell. Control Syst., vol. 3, no. 4, pp. 409-441, 1999.

[5] D. Pomerleau, "RALPH: Rapidly Adapting Lateral Position Handler," in Proc. IEEE Intell. Vehicles Symp., Detroit, MI, 1995, pp. 506-511.

[6] R. Rajamani, H.-S. Tan, B. K. Law, and W.-B. Zhang, "Demonstration of integrated longitudinal and lateral control for the operation of automated vehicles in platoons," IEEE Trans. Control Syst. Technol., vol. 8, no. 4, pp. 695-708, Jul. 2000.

[7] J. Farrell, H.-S. Tan, and Y. Yang, "Carrier phase GPS-aided INS based vehicle lateral control," Trans. ASME, J. Dyn. Syst. Meas. Control, vol. 125, no. 3, pp. 339-353, Sep. 2003.

[8] H.-S. Tan, B. Bougler, J. A. Farrell, and Y. Yang, "Automatic vehicle steering controls: DGPS/INS and magnetic markers," in Proc. Amer. Control Conf., Denver, CO, Jun. 2003, pp. 60-65.

[9] H.-S. Tan, B. Bougler, and W.-B. Zhang, "Automatic steering based on roadway markers-From highway driving to precision docking," Veh. Syst. Dyn., vol. 37, no. 5, pp. 315-339, Mar. 2002.

[10] T. Hessburg and M. Tomizuka, "Fuzzy logic control for lateral vehicle guidance," IEEE Control Syst. Mag., vol. 14, no. 4, pp. 55-63, Aug. 1994.

[11] S. Chaib, M. S. Netto, and S. Mammar, " $H_{\infty}$, adaptive, PID and Fuzzy control: A comparison of controllers for vehicle lane keeping," in Proc. IEEE Intell. Veh. Symp., Jun. 2004, pp. 139-144.

[12] M. A. Sotelo, F. J. Rodriguez, and L. Magdalena, "VIRTUOUS: Vision based road transportation for unmanned operation on urban like scenarios," IEEE Trans. Intell. Transp. Syst., vol. 5, no. 2, pp. 69-83, Jun. 2004.

[13] U. Franke, D. Gavrila, S. Görzig, F. Lindner, F. Paetzold, and C. Wöhler, "Autonomous driving approaches downtown," IEEE Intell. Syst., vol. 13, no. 6, pp. 40-48, Nov. 1999.

[14] E. D. Dickmanns and A. Zapp, "A curvature based schema for improving road vehicle guidance by computer vision," in Proc. SPIE-Conf. Mobile Robots, Cambridge, MA, 1986, vol. 727, pp. 161-168.

[15] T. Endo, K. Iwazaki, and Y. Tanaka, "Development of reverse parking assist with automatic steering," in Proc. ITS Congr., Madrid, Spain, Nov. 2003

[16] T. Hessburg and M. Tomizuka, "Fuzzy logic control for lane change maneuvers in lateral vehicle guidance," Univ. Calif., Berkeley, CA, Calif. PATH Working Paper UCB-ITS-PWP-95-13, Oct. 1995. 
[17] D. Swaroop and S. M. Yoon, "The design of a controller for a following vehicle in an emergency lane change maneuver," Univ. Calif., Berkeley, CA, Calif. PATH Working Paper UCB-ITS-PWP-99-3, Feb. 1999.

[18] J. K. Hedrick, D. H. McMahon, V. K. Naredran, and D. Swaroop, "Longitudinal vehicle controller design for IVHS systems," in Proc. Amer. Conf., 1991, pp. 297-303.

[19] R. Horowitz, C. W. Tan, and X. Sun, "An efficient lane change maneuver for platoons of vehicles in an automated highway system," Univ. Calif., Berkeley, CA, Calif. PATH Res. Rep. UCB-ITS-PRR-2004-16, May 2004.

[20] C. Hatipoglu, U. Özgüner, and K. A. Redmill, "Automated lane change controller design," IEEE Trans. Intell. Transp. Syst., vol. 4, no. 1, pp. $13-$ 22, Mar. 2003.

[21] H. Jula, E. B. Kosmatopoulos, and P. Ioannou, "Collision avoidance analysis for lane changing and merging," IEEE Trans. Veh. Technol., vol. 49, no. 6, pp. 2295-2308, Nov. 2000.

[22] S. Kato, S. Tsugawa, K. Tokuda, T. Matsui, and H. Fujiri, "Vehicle control algorithms for cooperative driving with automated vehicles and intervehicle communications," IEEE Trans. Intell. Transp. Syst., vol. 3, no. 3, pp. 155-161, Sep. 2002.

[23] M. Sugeno and M. Nishida, "Fuzzy control of a model car," Fuzzy Sets Syst., vol. 16, no. 2, pp. 103-113, Jul. 1985.

[24] J. E. Naranjo, C. González, R. García, T. de Pedro, and R. E. Haber, "Power-steering control architecture for automatic driving," IEEE Trans. Intell. Transp. Syst., vol. 6, no. 4, pp. 406-415, Dec. 2005.

[25] J. E. Naranjo, C. González, R. García, and T. de Pedro, "ACC+Stop\&Go maneuvers with throttle and brake fuzzy control," IEEE Trans. Intell. Transp. Syst., vol. 7, no. 2, pp. 213-225, Jun. 2006.

[26] R. García, T. de Pedro, J. E. Naranjo, J. Reviejo, and C. González, "Frontal and lateral control for unmanned vehicles in urban tracks," in Proc. IEEE Intell. Vehicles Symp., Versailles, France, 2002, pp. 583-588.

[27] J. Michon, "A critical view of driver behavior models: What do we know, what should we do?" in Human Behavior and Traffic Safety, L. Evans and R. Schwing, Eds. New York: Plenum, 1985.

[28] J. M. Mendel, "Fuzzy logic systems for engineering: A tutorial," Proc. IEEE, vol. 83, no. 3, pp. 345-377, Mar. 1995.

[29] E. H. Mamdani, "Application of fuzzy algorithms for control of a simple dynamic plant," Proc. Inst. Elect. Eng., vol. 121, no. 12, pp. 1585-1588, 1974.

[30] M. Sugeno, Industrial Applications of Fuzzy Control. Amsterdam, The Netherlands: North-Holland, 1985.

[31] M. Sugeno, "On stability of fuzzy systems expressed by fuzzy rules," IEEE Trans. Fuzzy Syst., vol. 7, no. 2, pp. 201-224, Apr. 1999.

[32] M. Sugeno, H. Winston, I. Hirano, and S. Kotsu, "Intelligent control of an unmanned helicopter based on fuzzy logic," in Proc. 51st Annu. Forum Amer. Helicopter Soc., Houston, TX, May 1995, pp. 791-803.

[33] P. Salgado and J. B. Cunha, "Greenhouse climate hierarchical fuzzy modelling," Control Eng. Pract., vol. 13, no. 5, pp. 613-628, May 2005.

[34] G. Vachkov and T. Fukuda, "Structured learning and decomposition of fuzzy models for robotic control applications," J. Intell. Robot. Syst., vol. 32, no. 1, pp. 1-21, Sep. 2001.

[35] T. Takagi and M. Sugeno, "Fuzzy identification of systems and its applications to modeling and control," IEEE Trans. Syst. Man, Cybern., vol. SMC-15, no. 1, pp. 116-132, Jan./Feb. 1985.

[36] W.-K. Chen, Linear Networks and Systems. Belmont, CA: Wadsworth, 1993, pp. 123-135.

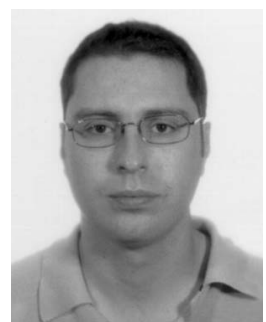

José E. Naranjo was born in Orense, Spain, in 1975. $\mathrm{He}$ received the B.E. and M.E. degrees in computer science engineering in 1998 and 2001, respectively, and the Ph.D. degree in computer science in 2005 from the Polytechnic University of Madrid (UPM), Madrid, Spain.

From 1999 to 2007, he was with the Industrial Computer Science Department, Instituto de Automática Industrial, Madrid. He is currently an Associate Professor with the Computer Science School, Universidad Politécnica de Madrid. His research interests include intelligent systems, fuzzy logic control, and ITS.

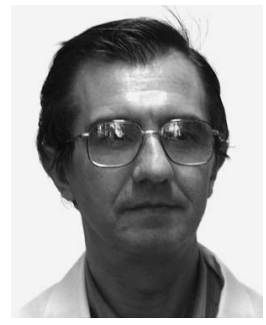

automation projects.

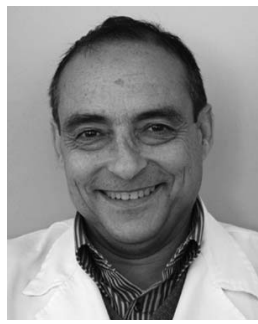

Ricardo García received the Ph.D. degree in physics from Bilbao University, Bilbao, Spain, in 1974.

He was the Founder of the Instituto de Automática Industrial, Spanish National Research Council, Madrid, Spain, where he has been working in intelligent robotics.

Dr. García was the recipient of the "Barreiros" Research on Automotive Field Prize in 2002 for his AUTOPIA project on intelligent transport systems.

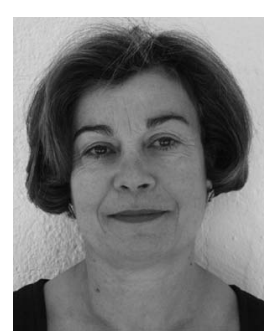

Teresa de Pedro received the Ph.D. degree in physics from the Universidad Complutense de Madrid, Madrid, Spain, in 1976.

Since 1971, she has been working on artificial intelligence applied to automation with the Instituto de Automática Industrial, Spanish National Research Council, Madrid. She is currently interested in fuzzy models for unmanned vehicles. She is the Head of a Spanish team that is involved with the Integration of Sensors to Active Aided Conduction project. 